\title{
A transcription factor, TFIS, interacts with both the promoter and enhancer of the Xenopus rRNA genes
}

\author{
Marietta Dunaway \\ Department of Molecular and Cell Biology, University of California, Berkeley, California 94720 USA
}

\begin{abstract}
An activity that binds sequence specifically to the enhancer of the Xenopus laevis rRNA genes has been highly purified. This activity stimulates transcription of coinjected rRNA templates in Xenopus oocytes and has been named TFIS, as it binds to the enhancer sequences within the intergenic spacer. In addition to its enhancer binding activity, TFIS binds to the promoter of the Xenopus rRNA genes, as predicted by models for enhancer action. DNase I footprinting on promoter mutants suggests that there are three TFIS-binding sites between -70 and $\mathbf{- 2 4 0}$ and that TFIS binding is unusually tolerant of mutations. The large region of protein-DNA interaction and the occurrence of DNase I enhancements at integral multiples of the helical repeat are consistent with the promoter and enhancer DNA wrapping around TFIS.
\end{abstract}

[Key Words: Xenopus rRNA; transcription factors; enhancer; DNA wrapping]

Received April 17, 1989; revised version accepted August 23, 1989.

The intergenic spacer of the Xenopus laevis rRNA genes has been studied in detail because of its ability to augment transcription in a manner that is relatively insensitive to position and orientation (Busby and Reeder 1983; Moss 1983; Reeder et al. 1983; Labhart and Reeder 1984). Although the spacer is composed of three distinct classes of repeated sequence elements, only the 60/81-bp repeats have been shown to have enhancer characteristics (Fig. 1). The activity of the enhancer repeats can be assayed by template competition in oocytes. Two transcription templates are coinjected; these two templates have identical promoters but one also contains spacer sequences in addition to the promoter. Under these circumstances, the 60/81-bp repeats confer a 20-fold advantage in transcriptional activation (Reeder et al. 1983). The Xenopus rRNA gene spacer is the best-characterized RNA polymerase I enhancer, but rat and yeast spacers also influence rRNA transcription (Elion and Warner 1984; Cassidy et al. 1986). The activity of spacers in these systems, together with the similar structures of the human, mouse, and Xenopus promoters, suggests a common mechanism of action for the rRNA genes (Grummt et al. 1986; Haltiner et al. 1986; Henderson and Sollner-Webb 1986; Labhart and Reeder 1986; Learned et al. 1986; Miller et al. 1985; McStay and Reeder 1986).

An intriguing feature of the 60/81-bp repeats is the extent of sequence identity, $\sim 80 \%$, between the repeats and the sequences in the promoter from about -80 to -120 (Boseley et al. 1979; Sollner-Webb and Reeder 1979). We proposed a model in which the spacer enhancer and the promoter require a common protein for activity. This model is based on the oocyte injection experiments described above and on the similarity between the spacer and promoter sequences. Presumably the protein interacts with both spacer and promoter in a sequence-specific manner. Support for this model comes from the result that transcription from a plasmid containing only the promoter is inhibited by coinjection with a plasmid containing only spacer repeats (Labhart and Reeder 1984).

Proof that the same protein actually does interact with the spacer and promoter sequences requires the purification and characterization of the protein. This paper reports the purification of a transcription factor, TFIS, that binds in a site-specific manner to both the spacer enhancer and to the promoter of the rRNA genes. On injection into oocytes, the purified protein stimulates transcription. TFIS binds to the sequences common to both enhancer and promoter, but its interaction with both enhancer and promoter extends beyond these sequences. Footprinting experiments on linker scanning (LS) mutants of the promoter indicate that the promoter contains multiple TFIS-binding sites. The periodicity of DNase I enhancements in the footprint and the large region of DNA that interacts with TFIS suggest that the DNA wraps around the protein.

\section{Results}

Purification of a spacer enhancer-binding activity

To identify the putative enhancer-binding factor, a DNase I footprinting assay was employed /Galas and 


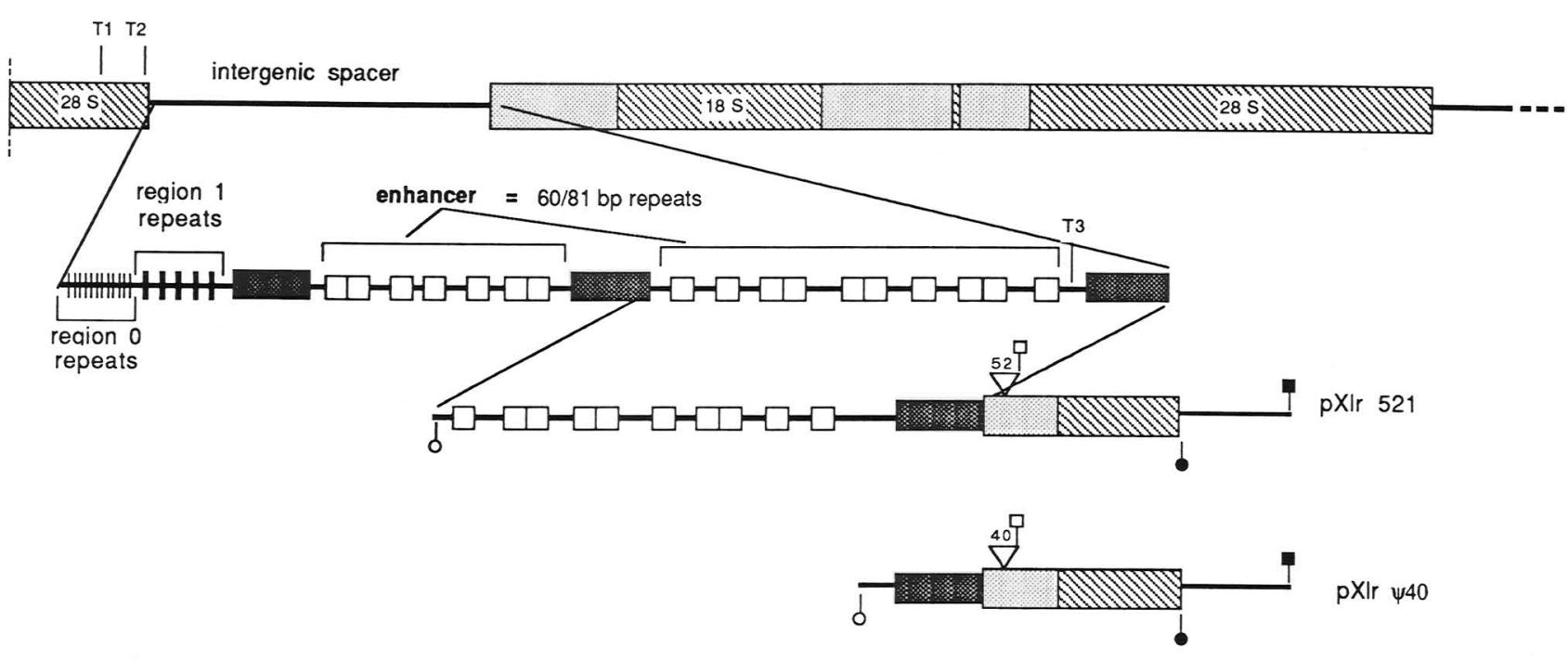

pXIr 60.81

pXIr 60

Figure 1. Structure of the Xenopus rRNA gene and spacer. A schematic representation of the Xenopus rRNA gene and spacer is shown. White boxes indicate 60-bp repeats; thick horizontal black lines between the repeats represent 21 -bp repeat sequences. Other classes of repeated sequence are represented by vertical lines and are labeled. Dark stippled rectangles represent promoters. Hatched regions within the gene indicate sequences that are present in the mature RNA products. T1 is a processing site, whereas $\mathrm{T} 2$ and $\mathrm{T} 3$ indicate the position of sequences necessary for 3 '-end formation. The transcription templates used in this study have been described in detail previously (Reeder et al. 1983); the insertion that distinguishes their transcripts is indicated with an open inverted triangle

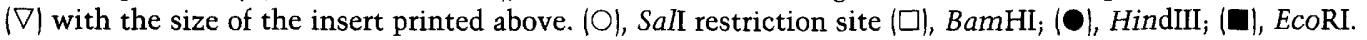

Schmitz 1978), using a probe derived from the spacer enhancer. Nuclei were isolated from Xenopus tissue culture cells and used as the starting material for the purification. The proteins from a salt eluate of the nuclei were precipitated with ammonium sulfate and applied to a Sephacryl S-300 column. An enhancer-specific binding activity was identified (data not shown). A specific DNA affinity column was prepared by linking the naturally occurring cluster of 10 enhancer repeats to Sepharose 4B-CL (Kadonaga and Tiian 1986; Rosenfeld and Kelly 1986). The peak fractions from the Sephacryl column were pooled and chromatographed on the enhancer column in the presence of $\sim 100$-fold excess of poly[d(A-T)]. Elution of the enhancer-binding activity by salt steps was again monitored by DNase I footprinting, and the enhancer-binding activity profile and a silver-stained SDS-polyacrylamide gel of the polypeptides present in the eluted fractions from the second round of purification on the DNA affinity column are shown in Figure 2.

Three major silver-staining polypeptides copurify with the enhancer-binding activity eluting in the 0.5 and $0.6 \mathrm{M} \mathrm{KCl}$ salt steps: a doublet of $\sim 110 \mathrm{kD}$ and a polypeptide of $35 \mathrm{kD}$. A trace of BSA that was used to stabilize the activity during the second-round DNA affinity column is present in all of the column fractions. The $110-\mathrm{kD}$ and the $35-\mathrm{kD}$ doublet protein consistently copurify with the binding activity. The relative concentrations of the $110-\mathrm{kD}$ doublet parallel that of the DNAbinding activity more closely than does the concentra- tion of the $35-\mathrm{kD}$ polypeptide. However, the $0.6 \mathrm{M} \mathrm{KCl}$ fraction, which contains more of the $35-\mathrm{kD}$ protein, was much more stable to repeated freezing and thawing (data not shown). Estimates of the concentrations of the protein and DNA binding template indicate that they are present in approximately stoichiometric amounts in the footprinting reactions. This corresponds to a maximum dissociation constant of $10^{-9} \mathrm{M}$. On the basis of these estimates of concentration and by use of the footprinting assay as a measure of activity, the purification of these proteins is on the order of 20,000 -fold. Further separation of these three polypeptides has not been successful, nor has DNA binding activity been recovered from SDS-polyacrylamide gels.

\section{Enhancer-binding activity is a transcription factor}

The transcriptional activity of the highly purified enhancer-binding activity was assayed by coinjection of protein and transcription templates into Xenopus oocytes. Because only a portion of the injected DNA templates is transcriptionally activated, coinjection of a transcription factor will increase the total amount of transcription if other essential proteins are not limiting. By using two transcription templates, one having only a promoter and the second having a promoter plus enhancer (Fig. 1), both total transcriptional stimulation and the relative activity of the enhancer with respect to the promoter can be assayed simultaneously. The tran- 


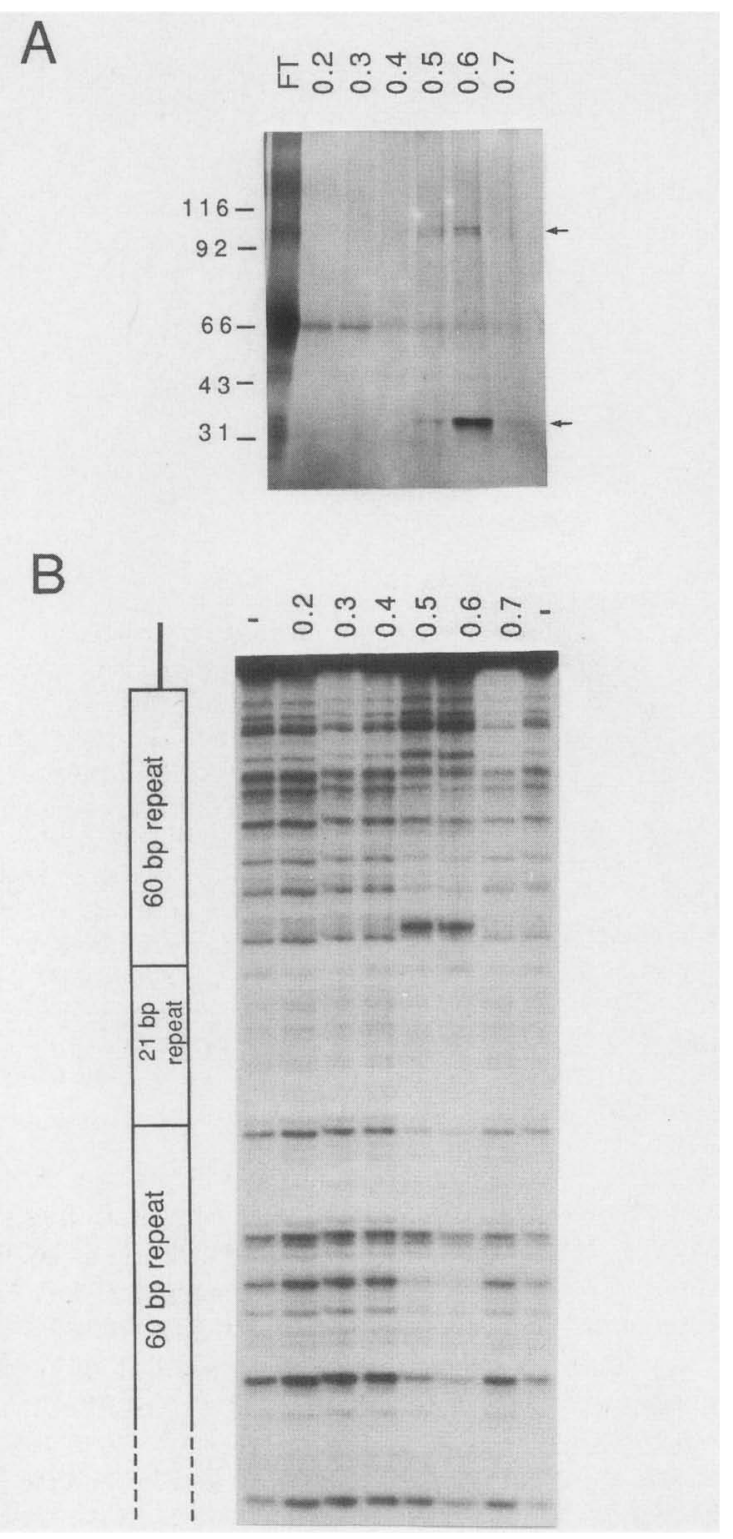

Figure 2. Elution of an enhancer-binding activity from a DNA affinity column. The $S-300$ column fractions containing the enhancer-binding activity were pooled, poly[d(A-T)] was added, and the protein was loaded onto a specific DNA affinity column. Protein was eluted in $100 \mathrm{~mm} \mathrm{KCl} \mathrm{steps,} \mathrm{as} \mathrm{indicated.}$ (A) Samples from the second round of purification from the enhancer-Sepharose column were TCA-precipitated and electrophoresed on an SDS-polyacrylamide gel containing an acrylamide gradient from $7.5 \%$ to $20 \%$. The gel was silver-stained. $(B)$ The footprinting activity of each fraction was assayed using a probe derived from pXlr60.81, which was labeled on the bottom strand (See Fig. 1).

scripts from these two templates can be distinguished from each other and from those from endogenous genes by using an S1 nuclease assay (Reeder et al. 1983; Labhart and Reeder 1985).

Coinjection of the enhancer-binding activity with the transcription templates stimulates transcription from both enhancer and promoter containing plasmids so that the ratio between the two is maintained (Fig. 3A). Densitometry of appropriate autoradiographic exposures indicates that this stimulation is fivefold at the highest protein level tested and that the increase in transcription for the two concentrations of protein tested is roughly linear. Figure 3B shows a blot of the injected DNA in this experiment; neither variation in injected DNA concentration nor variation in recovery of nucleic acid can be responsible for the stimulation of transcription observed when the enhancer-binding proteins are injected. Therefore, the highly purified enhancer-binding activity is a transcription factor; hence, the name TFIS is proposed because the proteins bind to the enhancer sequences within the intergenic spacer.

\section{Analysis of TFIS binding to the enhancer repeats}

DNase I footprinting of TFIS binding to a subclone of the spacer that contains two 60 -bp repeats and one 21 -bp repeat is shown in Figure 4. TFIS protects essentially all of an 81-bp unit consisting of one 60-bp repeat and the 21-bp repeat. The position of the 60-bp repeat in this binding unit is to the right of the 21-bp repeat with respect to transcription initiation. In addition, the remaining 60-bp repeat in this subclone also shows changes in DNase I digestion pattern. Many nucleotides in the region of interaction are completely protected from DNase I, but some regions of the DNA are still partially accessible to the enzyme. One of the most striking features of the footprints is the appearance of enhanced DNase I cleavage sites on both strands of the DNA. These enhancements are indicated with arrowheads in Figure 4, and the sequence at the enhanced cleavage sites is shown in Figure 6, below. The enhancement on the bottom strand of the 60-bp repeat (14 bases from the 2l-bp repeat) is particularly prominent and can be detected even when TFIS is present at low concentrations. Although the prominence of the enhancements is affected by protein concentration, salt concentration, extent of DNase I digestion, the probe preparation, and other parameters (cf. Figs. 2B and 4B), the position of the enhanced DNase I cleavage sites is very reproducible. The difference in appearance of Figures 2 and $4 \mathrm{~B}$ is also affected by how the gel was run because the DNase I ladder in Figure 2 corresponds to the upper two-thirds of the gel in Figure 4B. Binding to the left $60-\mathrm{bp}$ repeat is noticeably weaker than binding to the 81 -bp unit and seems to be independent, as higher concentrations of TFIS are necessary to protect this second 60 -bp repeat. This concentration dependence is particularly evident in Figure 4A. Titrations of TFIS binding to a single $60-\mathrm{bp}$ repeat also show that higher concentrations of TFIS are necessary for protection of the 60-bp repeat (Fig. 4C). Therefore, although a single 60 -bp repeat is sufficient to support TFIS binding, TFIS has a stronger interaction with a 60 -bp repeat flanked by a 21 -bp element to its left. Identification of a consensus in both the 60- and the 21-bp repeat (see Discussion and Fig. 8, below) suggests that this stronger interaction may occur because there are two TFIS-binding sites in the 81-bp unit. Alterna- 

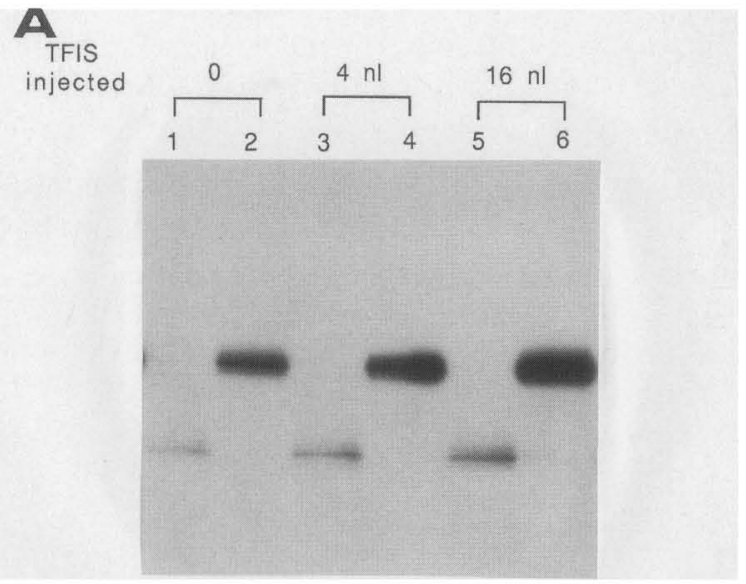

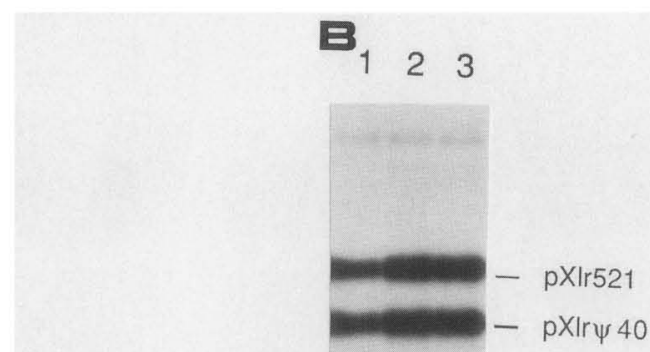

Figure 3. Transcriptional activity of the TFIS proteins microinjected into Xenopus oocytes. In all cases, both pXlr 440 and pXlr521 were coinjected with protein. After injection of the templates, oocytes were allowed to accumulate transcripts for $18 \mathrm{hr}$ before total nucleic acid was prepared from these oocytes. The two transcripts were assayed by S1 probes specific for each transcript. The template containing a 40-bp insertion (promoter) yields a 50-bp S1-protected fragment, and template containing a 52-bp insertion (enhancer) yields a 62-bp protected fragment. Protein samples were prepared for injection as described in Methods. In all cases, injection buffer contained $0.1 \mathrm{mg} / \mathrm{ml} \mathrm{BSA.} \mathrm{(A)} \mathrm{Three} \mathrm{injection} \mathrm{samples} \mathrm{were} \mathrm{prepared.} \mathrm{(Lanes} 1$ and 2) $8 \mu \mathrm{l}$ injection buffer, $1 \mu \mathrm{l} 0.5 \mathrm{mg} / \mathrm{ml} \alpha$-amanitin, $1 \mu 10.5 \mathrm{mg} / \mathrm{ml}$ DNA stock containing pXlr $\psi 40$ and pXlr521 in equal molar ratios: (lanes 3 and 4) $2 \mu l$ TFIS, $6 \mu l$ injection buffer, $1 \mu l$ $\alpha$-amanitin, $1 \mu$ l DNA; (lanes 5 and 6) $8 \mu$ l TFIS, $1 \mu \mathrm{l} \alpha$-amanitin, $1 \mu$ l DNA mix. Each oocyte was injected with $20 \mathrm{nl}$ of sample, and the labels indicate the amount of TFIS in this $20 \mathrm{nl}$. Lanes 1,3, and 5 show assays for the transcripts from the promoter-containing plasmid. Lanes 2, 4, and 6 show assays for transcripts from the enhancer plasmid. The activity of the enhancer is measured as the ratio of transcripts, so adjacent lanes should be compared. $(B)$ A portion of the same samples used in the transcription assays was digested with EcoRI and electrophoresed on an agarose gel. The gel was blotted, and the blot was hybridized with a labeled probe prepared from the minigene. (Lane 1) Injection of pXlr 440 and pXlr521 with injection buffer, corresponding to lanes 1 and 2 in $A$; (lane 2) same as lanes 3 and 4 in $A$; (lane 3 ) same as lanes 5 and 6 in $A$.

tively, one TFIS-binding unit may interact with both the 60- and 21-bp sequences.

\section{Analysis of TFIS binding to wild-type and mutant promoters}

TFIS was tested for its ability to bind to the promoter of the Xenopus rRNA genes by DNase I footprinting. Similar to its binding to the enhancer, the TFIS footprint contains several enhancements and protected regions in the promoter [Fig. 5A,B; Fig. 7 (WT), below]. Enhancements appear in the region from -70 to -150 and again between -180 and -220 . The borders of the TFIS interaction around -220 cannot be determined precisely using this construction. A convenient landmark is the sequence from -80 to -120 , which is $80 \%$ identical to the enhancer repeats. The enhancements at the $5^{\prime}$ border of the region from -70 to -150 occur at -136 on the top strand and -158 on the bottom strand, in good agreement with the border of the essential promoter that was determined by deletion mapping (Moss 1982; Sollner-Webb et al. 1983). The second region in which enhancements occur, from -180 to -220 , contains a third TFIS-binding site. This third TFIS-binding site occurs in an interesting region of the promoter that is involved in both transcription termination and in the stability of the transcription complex (Labhart and Reeder 1985; McStay and Reeder 1986). This third binding site is separated from the other two by $30 \mathrm{bp}$ of sequence consisting entirely of $\mathrm{G}$ and $\mathrm{C}$ residues. There are some nucleotides in the GC region that are protected from DNase I (Fig. 6), but the area of protection is small, there are no enhancements, and no consensus sequence has been identified. The areas of protection may be the result of protein interaction with the TFIS-binding sites on either side of this GC sequence and do not appear to be an independent binding site. The extent of protection in this region is misleading in Figure 5B, because lane 5 is somewhat underdigested; the accessibility of this region to DNase I is more typical in Figure 7 (WT).

In addition to the TFIS-binding regions in the upstream region of the promoter, there is a TFIS-binding site that spans the transcription initiation site and extends into the coding region of the gene (Fig. 5A,B). Footprinting on the promoter deletion mutant $\Delta 5^{\prime}-26$ indicates that binding to the internal site is not dependent on the presence of the upstream promoter-binding sites characterized above (data not shown). The $3^{\prime}$ boundary of the promoter maps to +16 by oocyte injection, which would include a portion of this TFIS-binding site.

To define the TFIS-binding sites in the promoter in more detail, DNase I footprinting on a series of LS mutants was done. These mutants were prepared by Reeder and colleagues (1987), and their transcriptional properties have been studied both in vitro and by oocyte injection. Only the LS mutants having lesions between -42 and -83 were used in the binding experiments here. In addition to the LS mutants, TFIS binding to a T3 box mutant at -201 was also examined (McStay and Reeder 1986). This mutation decreases promoter activity in mu- 

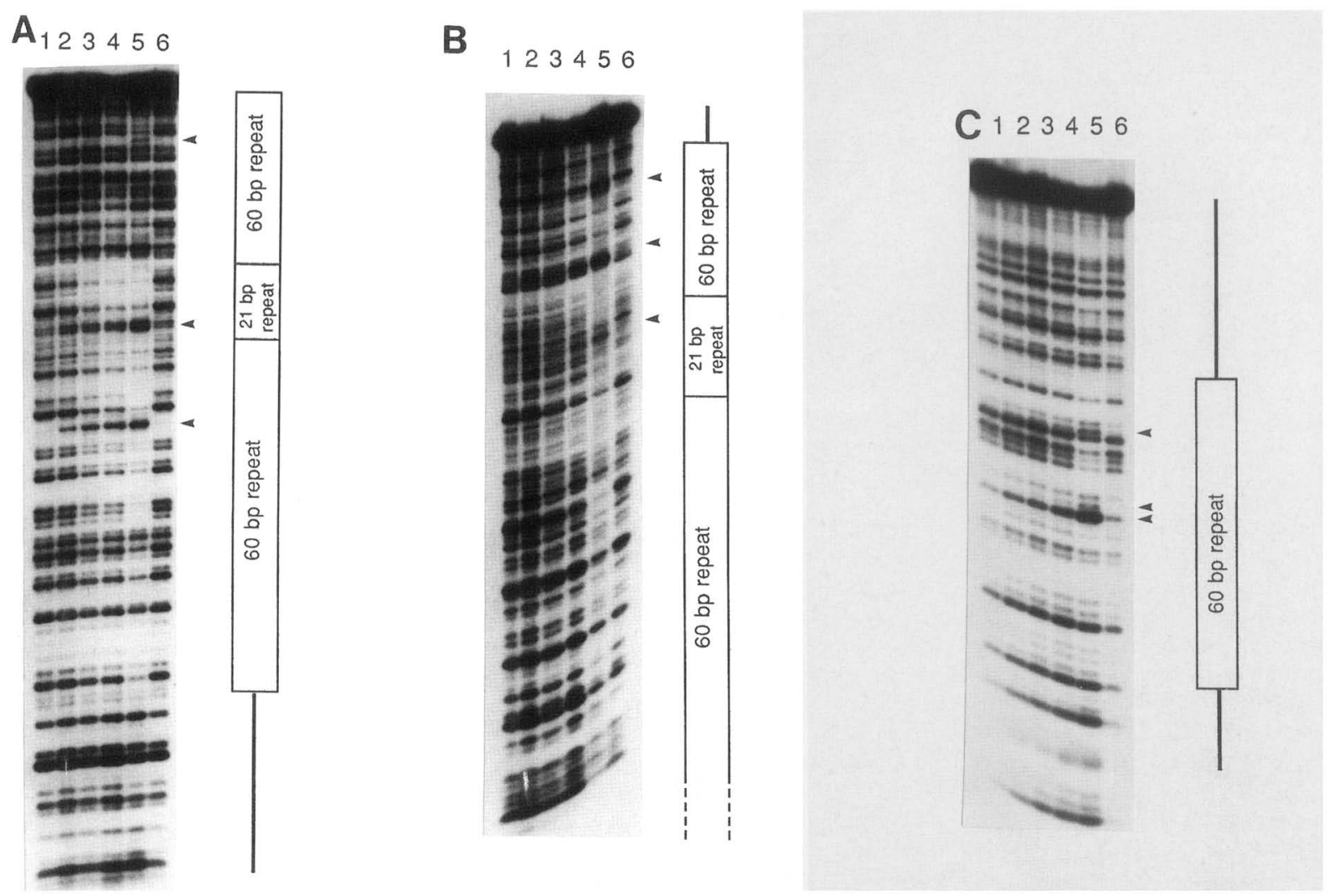

Figure 4. DNase I footprinting of TFIS on the enhancer repeats. Footprinting on pXlr60.81. The spacer subclone was cut with either EcoRI or HindIII in the polylinker, phosphatased, and labeled at the $5^{\prime}$ end with $\left[\gamma^{-32} \mathrm{P}\right] \mathrm{ATP}$, to visualize the top or bottom strand, respectively. The labeled probes were cut with an appropriate second enzyme to produce a probe labeled at only one end. The highly purified TFIS was incubated with 1-2 $\mathrm{ng}$ of the end-labeled probe for $10 \mathrm{~min}$ at room temperature under the conditions described in Methods. Major enhancements are indicated with arrowheads. Black lines indicate vector sequences. (A) Top strand. (Lanes 1 and 6 ) No protein; (lanes $2-5) 5 \mu l, 10 \mu l, 20 \mu l, 40 \mu l$ purified TFIS. $(B)$ Bottom strand. Lanes are identical with $A$. $(C)$ Footprinting on a single 60 -bp repeat. The clone pXlr60 was cut at the HindIII site in the polylinker of pUCl18 and end-labeled with $\left[\gamma^{-32} \mathrm{P}\right] \mathrm{ATP}$ to visualize the bottom strand. Lanes identical to $A$ and $B$.

tant promoter/wild-type promoter competition experiments.

Figure 7A shows TFIS binding to the top strand of the LS mutants under saturating protein concentrations. The DNase I footprints on these mutants were evaluated with respect to changes in areas of protection and changes in the strength and position of enhancements. The arrowheads indicate the same DNase I enhancements shown in Figure 5B, and the bars indicate the mutated sequences. Three of the mutants show clear defects in binding: LS $-142 /-133$, LS $-130 /-121$, LS $-111 /-102$. Mutants LS $-142 /-133$ and LS $-111 /-$ 102 also show the most extreme defects in transcription (Reeder et al. 1987); LS $-142 /-133$ is completely inactive in vitro and in the oocyte, whereas LS - 111/-102 shows only $3 \%$ of the in vitro activity of wild type and $1 \%$ activity in oocyte injection. LS $-130 /-121$ shows $20 \%$ of the transcriptional activity of wild type in vitro but only $7 \%$ wild-type activity in oocyte injection. In each case, only a portion of the TFIS promoter contacts are affected. In the TFIS footprint of mutant LS - 142/133 , the enhancement at -136 is eliminated, and the sequence between -138 and -119 is no longer pro- tected. It is interesting that there are changes in the DNase I digestion pattern of LS - 142/-133 DNA well outside the mutated region, which suggests that the DNA structure is changed. This raises the possibility that structural changes, as well as changes to primary sequence, may affect TFIS binding. The TFIS footprint of the bottom strand also loses the DNase I enhancement at -140 , and very little protection is observed between -150 and -119 (not shown). The TFIS footprint of mutant LS $-130 /-121$ has lost the DNase I enhancement at position -136 and shows decreased protection of sequences between -138 and -119 . The DNase I digestion pattern of the LS -130/-121 DNA also shows changes outside the mutated region compared with wild type. Footprinting on the bottom strand of this mutant (not shown) also shows no enhancements and decreased protection in the same region. The loss of enhancement in LS $-130 /-121$ is not due to sequence changes at the site of the enhancement. Both LS $-142 /-133$ and LS-130/-121 disrupt the same DNA-protein contacts, but the mutated sequences are 10 nucleotides apart, indicating that the TFIS-binding site is quite large. Mutant LS $-111 /-102$ has lost enhancements at -118 
A

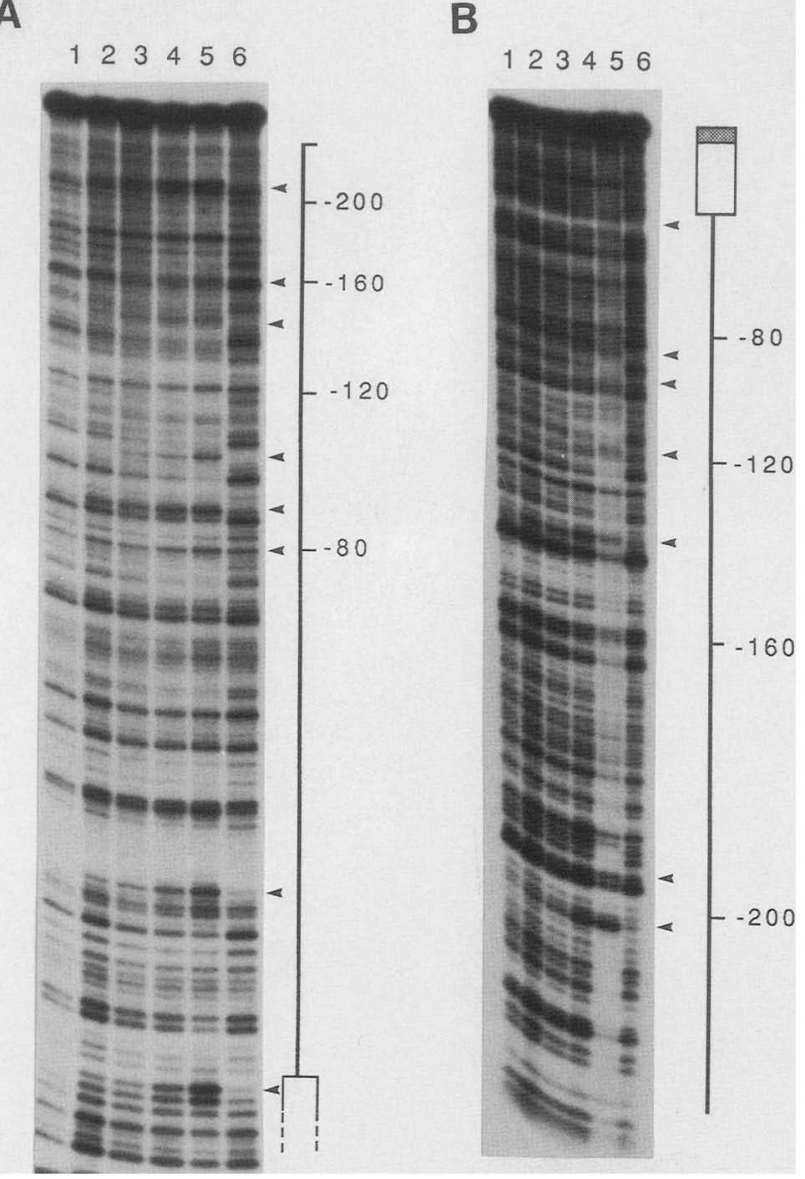

Figure 5. DNase I footprinting on the promoter of the Xenopus rRNA genes. pXlr 440 was cut either with SalI to label the top strand of the promoter or with $B a m H I$ in the inserted 40 -bp sequence to label the bottom strand. Black lines indicate promoter sequences, white boxes indicate sequence derived from the external transcribed spacer, and the stippled box indicates $28 \mathrm{~S}$ sequences that are part of the minigene construct pXlr 440 . Lanes are identical to those in Fig. 4. (A) Bottom strand; $(B)$ top strand.

and at -95 and has also lost protection in the immediate area of the enhancements. The bottom strand shows more extensive changes in protection and loss of the reciprocal enhancement at -100 (not shown). In this case, also, the sequence at the position of the usual DNase I enhancement is not changed. Because LS - 111/ -102 affects a different set of protein-DNA contacts from those of LS $-142 /-133$ and LS $-131 /-121$, it is likely that LS $-111 /-102$ is positioned in a second TFIS-binding site. By comparing the sequences from these and other binding sites, a consensus sequence for TFIS binding is proposed (Fig. 8).

Changes in the DNase I signature of TFIS on the mutants LS $-96 /-87$ and LS $-83 /-75$ were also observed. In the TFIS footprint of LS $-83 /-75$, the positions of the two enhancements usually found at -82 and -72 are both shifted so that the enhancements are closer together. Indeed, the enhancement that is shifted from
-82 to about -76 is located in a region that is normally protected from DNase I. The position of the enhancement that usually occurs at -82 is also shifted in the TFIS footprint of LS $-96 /-87$. The transcription data indicate that neither of these mutations is particularly deleterious, and LS $-83 /-75$ even shows an increase in transcriptional activity over wild type by oocyte injection (Reeder et al. 1987).

A similar binding experiment to that shown in Figure 7A was done with lower protein concentration, about half saturation, to determine whether more subtle differences in binding could be detected (not shown). None of the other mutants showed any obvious difference in ability to bind TFIS compared with wild type. The differences shown by LS $-142 /-133$, LS $-130 /-121$, and LS -111/-102 are essentially the same as those at higher protein concentration; no further protein-DNA interactions appear to be weakened.

A comparison of the differences in DNase I signature among wild type and the three LS mutants displaying binding defects that correlate with transcription defects is shown schematically in Figure 7B. The data suggest that the region between -70 and -160 of the promoter

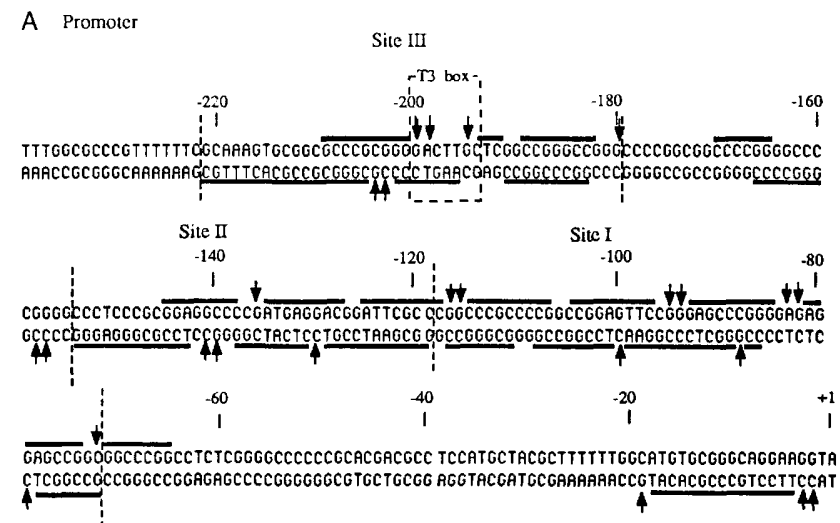

B pXlr60.81

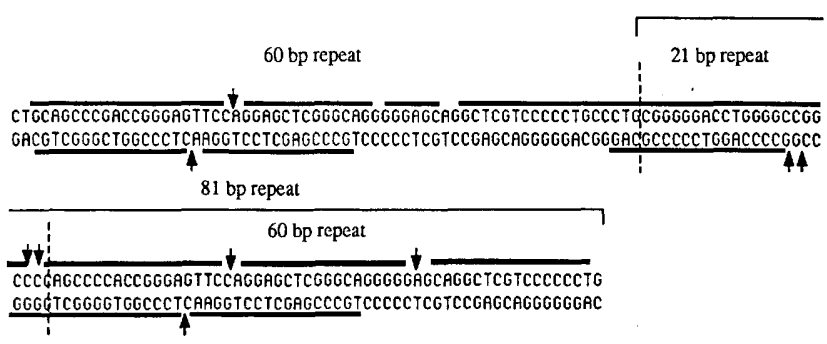

Figure 6. Summary of DNase I footprint data. Major enhancements are indicated with black arrows, and areas of protection are underlined with thick black lines. Sequences indicated as being protected may be either fully or partially protected. $(A)$ Promoter. The three TFIS-binding sites are labeled I, II, and III, and their suggested boundaries are indicated by vertical dashed lines. The T3 box is enclosed with a dashed line. $(B)$ The spacer subclone pXlr60.81. The $21-$ and $60-\mathrm{bp}$ repeats that act together as a unit are indicated with the bracket. 


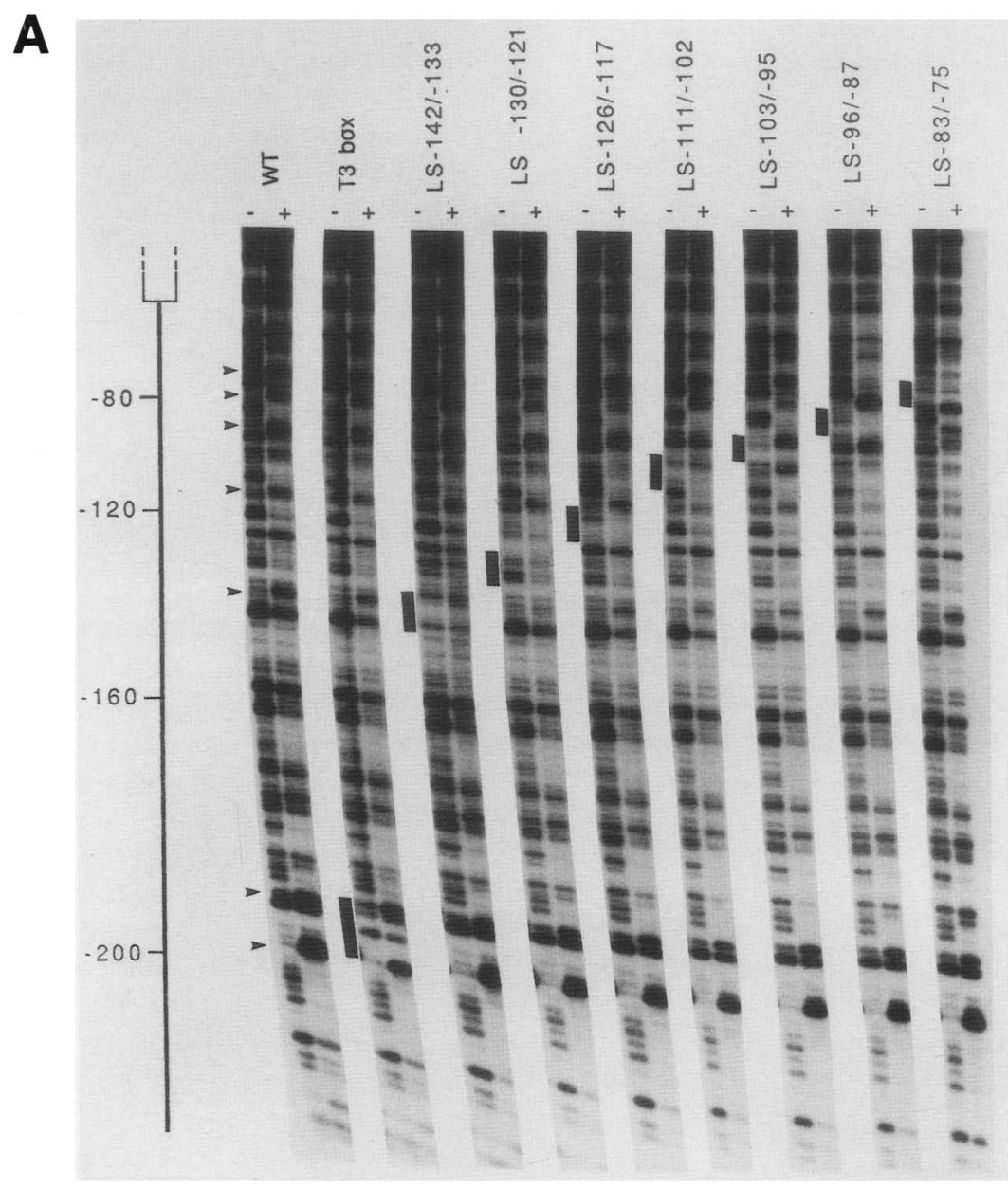

B

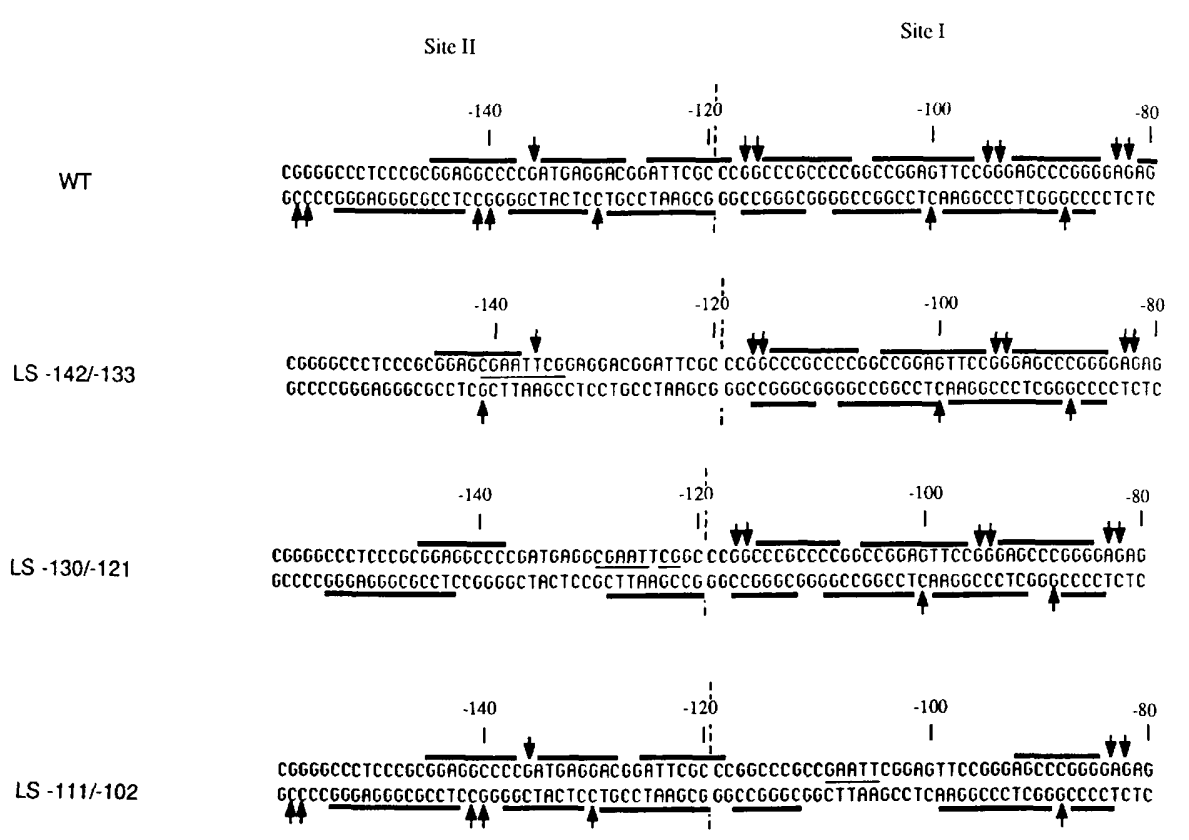

Figure 7. Footprinting on LS mutants of the promoter. Mutant and wild-type templates were labeled at the SalI site at -240, using polynucleotide kinase so that the top strand is labeled. $(A)$ Footprinting, using $40 \mu \mathrm{l}$ of highly purified TFIS. Black rectangles indicate the mutated sequence. Arrowheads mark the same enhancements as those in Fig. 5B. $1+$ and -1 With and without TFIS, respectively. (B) Summary of the changes in binding in those mutants showing significant differences from wild type. Symbols are as in Fig. 6 . 


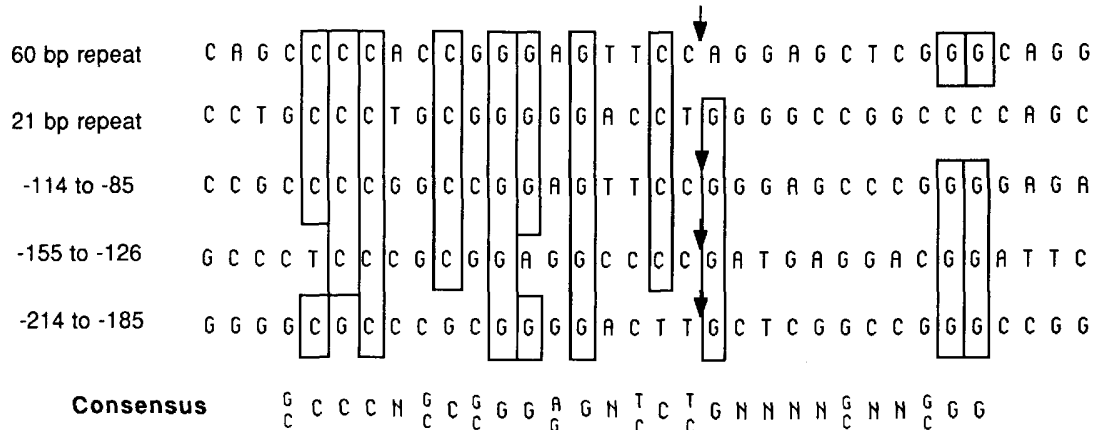

LS-142/-133 GC C C T C C C GCGGAGCG A A T TCGGAGGACG GA T T C

LS $-130 /-121$ GC C C T C C C GCG G A G G C C C C GATGAG G C G A A T T C G

LS-111/-102 C C GC CGAAT T C G GAG T T C C G G GAGC C C G G G GAG A
Figure 8. Consensus binding site for TFIS. The arrow marks the DNase I cleavage site used to align the sequences. In positions at which four of five binding sites are identical, only that base appears in the consensus sequence. These positions are boxed. At positions where all of the binding sites have either of only two bases, both are indicated. (N) No discernible conservation of primary sequence at that position in the four binding sites. contains two binding sites for TFIS. Because LS - 142/ 133 and LS - 130/-121 both affect the same proteinDNA contacts - although the mutated nucleotides are 13 nucleotides apart-it is inferred that the TFISbinding site spans both mutations. The third LS mutant in this group, LS $-111 /-102$, weakens a different set of contacts, suggesting that these contacts are contained within a second TFIS-binding site. Although it is formally possible that TFIS consists of two unequal binding domains and that binding to each is affected by different mutations, the identification of a consensus sequence from these two regions is consistent with two TFISbinding sites.

\section{Discussion}

A protein that binds specifically to both the promoter and enhancer of the $X$. laevis rRNA genes has been highly purified. Coinjection of the highly purified protein with rRNA templates into oocytes stimulates transcription fivefold at the highest protein concentration tested, and the proportional transcriptional advantage of templates having an enhancer is maintained. The DNase I signature of TFIS is not typical of eukaryotic transcription factors. TFIS footprints exhibit dramatic DNase I enhancements, and the sequence between these enhanced cleavage sites may be fully or partially protected from DNase I.

Both the position and the nature of the promoter TFIS footprints, including binding to the region spanning the transcription initiation start site, are similar to those of the human UBFl and SLl proteins (Bell et al. 1988). These workers have shown that UBF1 interacts with both the upstream control element (UCE) and core promoter sequences and that UBF binding to the core sequences is involved in transcription. Purified UBF1 migrates as a doublet of 94 and $97 \mathrm{kD}$ on an SDS-protein gel. The similar size of the doublet at $110 \mathrm{kD}$ copurifying with TFIS activity and the similarity of UBF1/SL1 and TFIS footprints suggest that part of the activity purified as TFIS is almost certainly the Xenopus equivalent of human UBF1.
In previous oocyte injection experiments, injecting increasing amounts of the two transcription templates increased the competition between the enhancer and the promoter (Reeder et al. 1983). This is consistent with the two sequences competing for the same protein. From those results, one predicts that enough protein could be injected to saturate all binding sites eventually and that competition between enhancer and promoter would then be relieved. In the TFIS injections, total transcription is stimulated, but the competitive difference between the two plasmids was maintained rather than being alleviated. After dialysis of TFIS for injection, only a small fraction of the footprinting activity remains. It therefore seems likely that insufficient protein was injected to saturate all spacer and promoter binding sites, and so, the spacer enhancer still confers a transcriptional advantage in the competition assay.

Footprinting on wild-type and LS mutants of the promoter suggests that the region between -80 and -150 contains two TFIS-binding sites, labeled I and II in Figure 6. A third TFIS-binding site spans the sequences from -180 to -220 . Although TFIS does bind to the region of the promoter that shares $80 \%$ sequence identity with the enhancer (site I), neither site II nor site III has the same extensive sequence identity. TFIS promoter-binding site III coincides with a complex region of the promoter containing a mosaic of signal sequences for termination and transcription complex stability (Labhart and Reeder 1986; McStay and Reeder 1986). Given the putative involvement of TFIS in enhancer function and the large number of TFIS-binding sites in the spacer, it seems rather unlikely that it is the critical protein for transcription termination. Probably TFIS-binding site III contributes to the stability of the promoter transcription complex. Alternatively, the phasing and structure of TFIS-binding sites could provide a structural cue for the polymerase to terminate.

The footprinting on promoter mutants suggests that there are few, if any, protein-protein contacts involved in binding to adjacent TFIS-binding sites in the promoter, as mutations in site II do not affect binding to site I, although there may be cooperativity involved in 
binding to the $81-\mathrm{bp}$ repeat. Interestingly, LS mutations in the TFIS promoter-binding site II cause larger decreases in transcriptional activity than those in binding site I (Windle and Sollner-Webb 1986; Reeder et al. 1987). Neither titrations nor binding competition assays (M. Dunaway, unpubl.) have yet revealed any large differences in the affinity of TFIS for the three promoterbinding sites. Furthermore, all the LS mutants affect transcription, but only three seem to alter TFIS binding. The remaining LS mutants may disrupt binding of an unidentified protein that interacts with these sequences, or alternatively, these mutations may affect the structure of the DNA and the resulting transcription complex.

Although TFIS can bind to a single 60 -bp repeat, the DNase I protection patterns clearly show that the protein also interacts with the 21-bp element. Titrations indicate that there is a substantial increase in affinity for the entire 81 -bp sequence, compared to only the 60 -bp repeat. The interactions with the 21-bp element are linked to the 60-bp repeat to its right. A partial consensus sequence is found within the $21-\mathrm{bp}$ repeat that extends into the upstream 60 -bp repeat, so it seems likely that there are two TFIS-binding sites per 81 -bp repeat. It is noteworthy that the distance between central pyrimidine tracts in the 21 - and $60-\mathrm{bp}$ repeats is $30 \mathrm{bp}$, whereas the distance is 41 bp between the two promoter-binding sites from -80 to -160 . This change in distance may explain why the combination of the two binding sites in the enhancer repeats increases TFIS affinity, as closer binding sites may facilitate protein-protein interaction. In both cases, however, the distance between sites is an integral multiple of the helical repeat. Data from enhancer mutants in which one of the two sites is mutated are necessary to determine unambiguously whether these two protein-binding sites interact in the enhancer but not in the promoter.

The footprinting results reported here are largely in agreement with the transcriptional results of LS and deletion mutants of the enhancer (Pikaard and Reeder 1988). Major interactions between TFIS and the 60-bp repeat occur in the A domain defined by these workers. However, Pikaard and Reeder concluded that the 21-bp repeat had no detectable contribution to enhancer activity, although it is shown here that TFIS clearly interacts with the $21-\mathrm{bp}$ repeat. This difference in the transcription and protein-binding results may be due to the construct used in the transcription study. The 81-bp repeat in that study was only $76 \mathrm{bp}$ in length. Although this construct contains most of the nucleotides in the consensus, when it is polymerized to form a block of enhancer repeats, the spacing between TFIS-binding sites will be changed. In addition, the footprinting data show that the 21 -bp repeat is linked to the 60 -bp repeat to its right, and in the mutant constructions prepared by Pikaard and Reeder, the 21-bp repeat is cloned with the 60 -bp repeat to the left.

To deduce a consensus sequence for TFIS binding, the promoter-binding sites and 60 -bp repeat-binding sites were aligned by their central DNase I enhancements
(Fig. 8). Four LS mutations that affect TFIS binding, LS $-142 /-133$, LS $-130 /-121$, LS $-111 /-102$, and LS $-96 /-87$, fall within the consensus. However, the mutations in LS $-83 /-75$ do not fall into the consensus sequence. The major enhancements occur immediately to the right of a central pyrimidine tract. To the left of these four pyrimidines are four purines, either GAGG or GGAG. To the left of the GAGG quartet is a GC-rich sequence. The consensus shown is the summary of those positions at which three out of four of the binding sites match and positions in which only two different nucleotides are found. The partial consensus site in the 21-bp repeat was identified by sequence comparison; a DNase I enhancement is not found at the position corresponding to the other four sites. One striking feature of this sequence, in general, is that it seems to alternate between short tracts of purines and pyrimidines on each strand. Because of the structural similarities in the sites and because some of the LS mutations cause large changes in DNase I digestion pattern outside the mutated nucleotides, it is worth considering whether the DNA structure as well as the primary sequence contribute to TFIS binding.

The TFIS DNase I signature, the unusually large region that TFIS contacts, and the tolerance for mutation within the binding site all suggest that the TFIS-DNA interactions are rather unusual and certainly different from most other transcription factors. The DNase I footprint is dominated by strong, regularly spaced, enhanced cleavage sites. Periodic enhancements are characteristic of DNA lying on a surface (for review, see Travers and Klug 1987). The DNA can be wrapped or bent around the surface of a protein, such as the nucleosome or Escherichia coli cAMP activator protein (Lutter 1978; Taniguchi et al. 1979|, or it can be lying against an inert surface (Rhodes and Klug 1980). To illustrate the periodicity of the DNase I enhancements, the positions of the enhanced DNase I cleavage sites in the promoter are

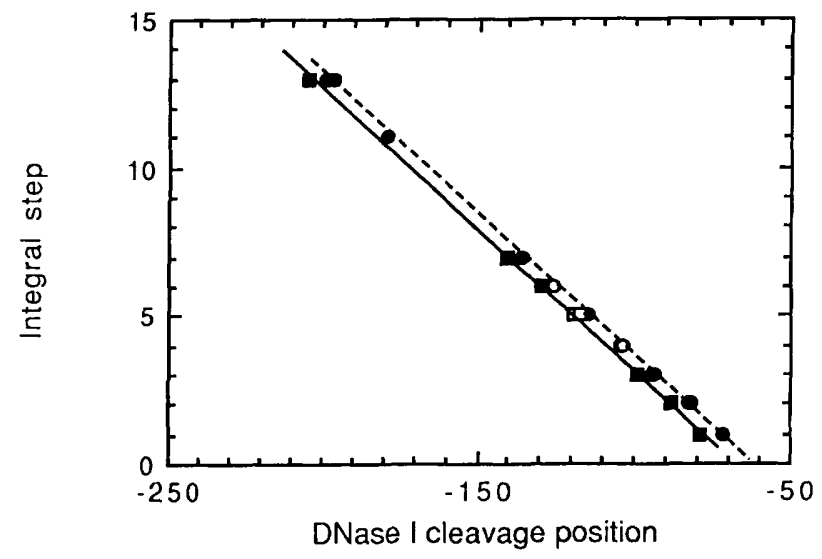

Figure 9. Periodicity of DNase I enhancements in the promoter. Integral steps of $10 \mathrm{bp}$ were assigned to the promoter sequence between -70 and -240 . The precise positions of DNase I enhanced cleavage sites were then plotted vs. the integral step in which they occur. (O) Top strand; ( $\mathbf{0}$ ) bottom strand. Positions at which DNase I cleavage is not inhibited by TFIS binding are indicated with open symbols. 
plotted against the integral step in which the cleavages occur (Fig. 9). The fit of the data to a line is an indication of the regularity of the enhancements, and the inverse slope will equal the helical repeat (Morrison and Cozzarelli 1981). The data from both strands of the promoter are an excellent fit to the line, and the helical repeat determined from the slope is $\mathbf{1 0 . 4}$ for both bottom and top strands of the promoter. The line for the bottom strand is displaced 4 nucleotides to the left of the top strand, as one would expect for a minor groove reagent like DNase I (Suck and Oefner 1986; Sluka et al. 1987). Furthermore, the plot indicates that the three TFIS-binding sites within the promoter are in phase. Because of the length of 60- and 21-bp repeats, TFIS binding to the enhancer will necessarily be in phase. The idea that the DNA of the promoter and enhancer is wound around TFIS is also consistent with the rather large size of the TFIS-binding site (Liu-Johnson et al. 1986).

\section{Methods}

\section{Preparation of spacer subclones}

Subclones of the spacer enhancer were prepared by digestion of the clone pXlr14F (Labhart and Reeder 1984) with PstI to generate the pXlr60.81 insert, or with PstI and SmaI to generate the single 60-bp repeat in pXlr60. Inserts were cloned into the vector pUCl18 (J. Vieira and J. Messing, unpubl.), and their content was determined by dideoxy sequencing (Biggin et al. 1983).

\section{Preparation of TFIS}

Nuclear extracts were prepared as described by Dignam et al. (1983), with the following modifications for amphibian cells. Xenopus A6 tissue culture cells were grown in roller bottles in $1 / 2 \times$ Leibowitz's medium supplemented with $10 \%$ FBS. Cells were scraped in their own medium and washed in homogenization buffer [10 mM HEPES (pH 7.6), $5 \mathrm{~mm} \mathrm{MgCl}, 10 \mathrm{~mm} \mathrm{KCl}$, $0.5 \mathrm{~mm}$ DTT, $0.1 \mathrm{~mm}$ PMSF]. The cells were resuspended in $1 / 2 \times$ homogenization buffer with NP-40 added to a final concentration of $0.2 \%$. Cells were dounced, and the proportion of broken cells was monitored by microscopy. The nuclear pellet was resuspended in $2 \times$ the volume of the original cell pellet in $20 \mathrm{~mm}$ Tris-Cl (pH 7.8), $1 \mathrm{~mm} \mathrm{DTT}, 5 \mathrm{~mm} \mathrm{MgCl}_{2}$, and $50 \%$ glycerol, frozen in an ethanol/dry-ice bath, and stored at $-80^{\circ} \mathrm{C}$.

Nuclei from two to five nuclei preparations $\left(10^{9}\right.$ to $\left.3 \times 10^{9}\right)$ were thawed, and the $\mathrm{KCl}$ concentration was adjusted to $0.5 \mathrm{M}$. The suspension was stirred for $30 \mathrm{~min}$ at $4^{\circ} \mathrm{C}$ and centrifuged. Ammonium sulfate was added to the supernatant to a final concentration of $50 \%$ saturation. The precipitate was collected by centrifugation and resuspended in $2 \mathrm{ml}$ of $20 \mathrm{mM}$ HEPES$\mathrm{K}^{+}(\mathrm{pH} 7.6), 5 \mathrm{mM} \mathrm{MgCl}_{2}, 1 \mathrm{mM}$ DTT, $0.1 \mathrm{mM}$ PMSF, and $15 \%$ glycerol. The protein was applied to a Sephacryl S-300 column (Pharmacia), and enhancer binding activity was monitored by footprinting. Fractions containing enhancer-binding activity were pooled, the salt concentration adjusted to $0.2 \mathrm{M} \mathrm{KCl}$, and poly[d(A-T) $] /$ poly[d(A-T)] was added to a final concentration of 2 $\mu \mathrm{g} / \mathrm{ml}$. The sample was applied to three $1-\mathrm{ml}$ specific DNA affinity columns that were prepared by cross-linking the enhancer repeats from pXlr14F to CNBr-activated Sepharose CL-4B (Kadonaga and Tjian 1986; Rosenfeld and Kelly 1986). TFIS activity was eluted by $1-\mathrm{ml}$ salt steps of $0.1 \mathrm{M}$ increments. The TFIS activity elutes at $\sim 0.45 \mathrm{M} \mathrm{KCl}$. The resulting active fractions were pooled, competitor added to $0.2 \mu \mathrm{g} / \mathrm{ml}$, BSA added to $0.1 \mathrm{mg} / \mathrm{ml}$, and the salt concentration adjusted to 0.2 M $\mathrm{KCl}$ by dilution for a second DNA column step. Further rounds of purification resulted in activity loss without any qualitative change in the polypeptides detected by silver staining.

DNase I footprinting

Plasmid DNA was cut by the restriction endonuclease specified in the figure legends, treated with calf alkaline phosphatase, and labeled with T4 polynucleotide kinase and [ $\left.\gamma^{-32} \mathrm{P}\right] \mathrm{ATP}$. An appropriate second restriction enzyme was used, and the footprinting probes were isolated on acrylamide gels. Probes were eluted into $0.3 \mathrm{~m}$ sodium acetate, $5 \mathrm{~mm}$ EDTA, and $1 \%$ SDS and subsequently phenol-extracted and ethanol-precipitated twice. Standard footprinting reactions for the experiments reported here were $100 \mathrm{mM} \mathrm{KCl}, 20 \mathrm{~mm}$ HEPES ( $\mathrm{pH}$ 7.6), $5 \mathrm{~mm} \mathrm{MgCl}_{2}$, $0.5 \mathrm{~mm}$ DTT, 20\% glycerol, $0.2 \mu \mathrm{g}$ poly[d(A-T)], $100 \mu \mathrm{g} / \mathrm{ml}$ BSA, and 1-2 ng template. TFIS was added last to the mixture and incubated for $5 \mathrm{~min}$ at room temperature prior to addition of DNase I. A stock solution of DNase I at $5 \mathrm{mg} / \mathrm{ml}$ was diluted into $100 \mathrm{~mm} \mathrm{MgCl} 2,100 \mathrm{~mm} \mathrm{CaCl}$, and 6-50 ng DNase I was added to the reaction, depending on the purity of the protein. DNase I digestions proceeded for $30 \mathrm{sec}$, after which the reaction was stopped, phenol-extracted, ethanol-precipitated, and run on a $7 \mathrm{M}$ urea, Tris-borate gel (Sanger and Coulson 1978). Sequence ladders were prepared by Maxam and Gilbert (1980). After electrophoresis, gels were dried and exposed to Kodak XAR-5 film with intensifying screens at $-80^{\circ} \mathrm{C}$ (Laskey and Mills 1977).

\section{Oocyte injection}

Because oocytes are extremely sensitive to glycerol, protein samples for injection were dialyzed against $20 \mathrm{~mm}$ HEPES- $\mathrm{K}^{+}$ (pH 7.6), $100 \mathrm{mM} \mathrm{NaCl}, 5 \mathrm{mM} \mathrm{MgCl}, 0.1 \mathrm{~mm} \mathrm{DTT}$, and $10 \%$ sucrose. BSA was added to the protein sample at a concentration of $100 \mu \mathrm{g} / \mathrm{ml}$. Injection mixes were prepared in which dialysis buffer with $0.1 \mathrm{mg} / \mathrm{ml}$ BSA was used to compensate for TFIS volume. Approximately $20 \mathrm{nl}$ of the sample was injected into the nucleus of the oocytes. Batches of 40-60 oocytes were injected for each experimental condition. Oocytes were incubated at $19^{\circ} \mathrm{C}$ overnight and were harvested the next day. Total nucleic acid was prepared from each batch of oocytes, and the accumulated transcripts from the injected templates were detected by S1 nuclease assay (Labhart and Reeder 1984). Gels were exposed to preflashed film and quantitated by scanning densitometry.

\section{Acknowledgments}

I thank Brian Markoff and Kathleen Scully for technical assistance. I also thank N. Cozzarelli, H. Echols, R. Harland, and A. Brivanlou for critical and insightful comments on the manuscript. This work was supported in part by a grant from the National Institute of General Medical Sciences and in part by grant NP-652 from the American Cancer Society.

\section{References}

Bell, S.P., M. Learned, H.-M. Jantzen, and R. Tjian. 1988. Functional cooperativity between transcription factors UBF1 and SL1 mediates human ribosomal RNA synthesis. Science 241: 1192-1197.

Biggin, M.D., T.J. Gibson, and G.F. Hong. 1983. Buffer gradient gels and ${ }^{35} \mathrm{~S}$ label as an aid to rapid DNA sequence determination. Proc. Natl. Acad. Sci. 80: 3963-3965.

Boseley, P., T. Moss, M. Machler, R. Portmann, and M.L. Birnstiel. 1979. Sequence organization of the spacer DNA in a 
ribosomal gene unit of X. laevis. Cell 17: 19-31.

Busby, S. and R.H. Reeder. 1983. Spacer sequences regulate transcription of ribosomal gene plasmids injected into Xenopus embryos. Cell 34: 989-996.

Cassidy, B.G., H.-F. Yang-Yen, and L.I. Rothblum. 1986. Transcriptional role for the nontranscribed spacer of rat ribosomal DNA. Mol. Cell. Biol. 6: 2766-2773.

Dignam, J.D., R.M. Lebovitz, and R.G. Roeder. 1983. Accurate transcription initiation by RNA polymerase II in a soluble extract from isolated mammalian nuclei. Nucleic Acids Res. 11: $1475-1489$.

Elion, E.A. and J.R. Warner. 1984. The major promoter element of rRNA transcription in yeast lies $2 \mathrm{~kb}$ upstream. Cell 39: 663-673.

Galas, D.J. and A. Schmitz. 1987. DNase footprinting: A simple method for the detection of protein-DNA specificity. $\mathrm{Nu}$ cleic Acids Res. 5: 3157-3170.

Grummt, I., A. Kuhn, I. Bartsch, and H. Rosenbauser. 1986. A transcription terminator located upstream of the mouse rDNA initiation site affects rRNA synthesis. Cell 47: 901 911.

Haltiner, M.M., S.T. Smale, and R. Tjian. 1986. Two distinct promoter elements in the human ribosomal RNA gene identified by linker scanning mutagenesis. Mol. Cell. Biol. 6: 227-235.

Henderson, S. and B. Sollner-Webb. 1986. A transcriptional terminator is a novel element of the promoter of the mouse ribosomal RNA gene. Cell 47: 891-900.

Kadonaga, J.T. and R. Tiian. 1986. Affinity purification of sequence specific DNA binding proteins. Proc. Natl. Acad. Sci. 83: 5889-5893.

Labhart, P. and R.H. Reeder. 1984. Enhancer-like properties of the $60 / 81 \mathrm{bp}$ elements in the ribosomal gene spacer of Xenopus laevis. Cell 37: 285-289.

- 1985. Xenopus ribosomal gene enhancers function when inserted inside the gene they enhance. Nucleic Acids Res. 13: 8999-9009.

- 1986. Characterization of three sites of RNA 3' end formation in the Xenopus ribosomal gene spacer. Cell 45: $431-$ 443.

Laskey, R.A. and A.D. Mills. 1977. Enhanced autoradiographic detection of ${ }^{32} \mathrm{P}$ and ${ }^{125} \mathrm{I}$ using intensifying screens and hypersensitized films. FEBS Lett. 82: 314-316.

Learned, R.M., T.K. Learned, M.M. Haltiner, and R.T. Tjian. 1986. Human rRNA transcription is modulated by the coordinate binding of two factors to an upstream control element. Cell 45: 847-857.

Liu-Johnson, H.-N., M.R. Gartenberg, and D.M. Crothers. 1986. The DNA binding domain and bending angle of E. coli CAP protein. Cell 47: 995-1005.

Lutter, L.C. 1978. Kinetic analysis of deoxyribonucleaseI cleavages in the nucleosome core: Evidence for a DNA superhelix. I. Mol. Biol. 124: 391-420.

Maxam, A.M. and W. Gilbert. 1980. Sequencing end-labeled DNA with base specific chemical cleavages. Methods Enzymol. 65: 499-560.

McStay, B. and R.H. Reeder. 1986. A termination site for Xenopus RNA polymerase I also acts as an element of an adjacent promoter. Cell 47: 913-920.

Miller, K.B., J. Tower, and B. Sollner-Webb. 1985. A complex control region of the mouse rRNA gene directs accurate initiation by RNA polymerase I. Mol. Cell. Biol. 5: 554-562.

Morrison, A. and N.R. Cozzarelli. 1981. Contacts between DNA gyrase and its binding site of DNA: Features of symmetry and asymmetry revealed by protection from nucleases. Proc. Natl. Acad. Sci. 78: 1416-1420.
Moss, T. 1982. Transcription of cloned Xenopus laevis ribosomal DNA microinjected into Xenopus oocytes, and the identification of an RNA polymerase I promoter. Cell 30: $835-842$.

-1983. A transcriptional function for the repetitive ribosomal spacer in Xenopus laevis. Nature 302: 223-228.

Pikaard, C.S. and R.H. Reeder. 1988. Sequence elements essential for function of the Xenopus laevis ribosomal DNA enhancers. Mol. Cell. Biol. 8: 4282-4288.

Reeder, R.H., J.G. Roan, and M. Dunaway. 1983. Spacer regulation of Xenopus ribosomal gene transcription: Competition in oocytes. Cell 35: 449-456.

Reeder, R.H., D. Pennock, B. McStay, J. Roan, E. Tolentino, and P. Walker. 1987. Linker scanner mutagenesis of the Xenopus laevis ribosomal gene promoter. Nucleic Acids Res. 15: 7429-7441.

Rhodes, D. and A. Klug. 1980. Helical periodicity of DNA determined by enzyme digestion. Nature 286: 573-578.

Rosenfeld, P.J. and T.J. Kelly. 1986. Purification of nuclear factor I by DNA recognition site affinity chromatography. I. Biol. Chem. 261: 1398-1408.

Sanger, F. and A. Coulson. 1978. The use of thin acrylamide gels for DNA sequencing. FEBS. Lett. 87: 107-110.

Sluka, J.P. S.J. Horvath, M.F. Bruist, M.I. Simon, and P.B. Dervan. 1987. Synthesis of a sequence-specific DNAcleaving peptide. Science 238: 1129-1132.

Sollner-Webb, B. and R.H. Reeder. 1979. The nucleotide sequence of the initiation and termination sites for ribosomal RNA transcription X. laevis. Cell 18: 485-499.

Sollner-Webb, B., J.A.K. Wilkinson, J. Roan, and R.H. Reeder. 1983. Nested control regions promote Xenopus ribosomal RNA synthesis by RNA polymerase I. Cell 35: 199-206.

Suck, D. and C. Oefner. 1986. Structure of DNase I at $2.0 \AA$ resolution suggests a mechanism for binding to and cutting DNA. Nature 321: 620-625.

Taniguchi, T., M. O'Neill, and B. de Crombrugghe. 1979. Interaction site of Escherichia coli cyclic AMP receptor protein on DNA of galactose operon promoters. Proc. Natl. Acad. Sci. 76: 5090-5094.

Travers, A.A. and A. Klug. 1987. The binding of DNA in nucleosomes and its wider implications. Phil. Trans. R. Soc. 317: $537-561$

Windle, J.J. and B. Sollner-Webb. 1986. Two distant and precisely positioned domains promote transcription of Xenopus laevis rRNA genes: Analysis with linker-scanning mutants. Mol. Cell. Biol. 6: 4585-4593. 


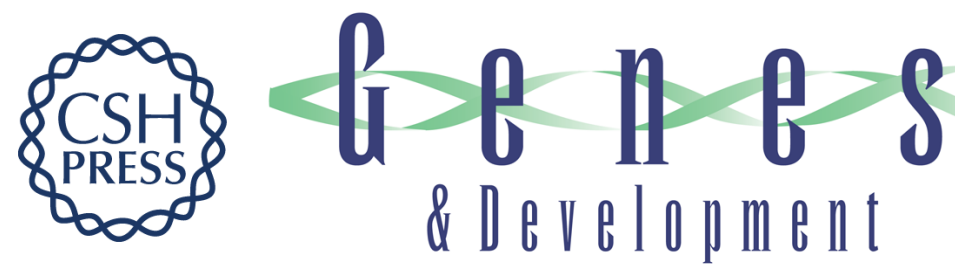

\section{A transcription factor, TFIS, interacts with both the promoter and enhancer of the Xenopus rRNA genes.}

M Dunaway

Genes Dev. 1989, 3:

Access the most recent version at doi:10.1101/gad.3.11.1768

References This article cites 38 articles, 12 of which can be accessed free at:

http://genesdev.cshlp.org/content/3/11/1768.full.html\#ref-list-1

License

Email Alerting

Service

Receive free email alerts when new articles cite this article - sign up in the box at the top right corner of the article or click here.

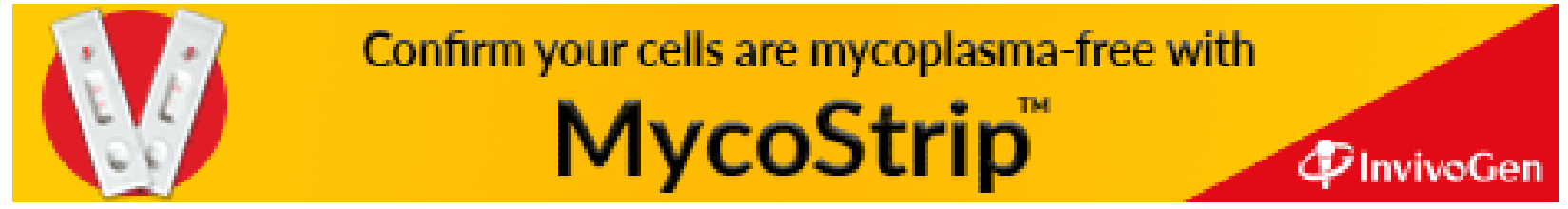

